\title{
Cognitive therapy reduces symptoms in people with recent onset post-traumatic stress disorder
}

Ehlers A, Clark DM, Hackmann A, et al. A randomized controlled trial of cognitive therapy, a self-help booklet, and repeated assessments as early interventions for posttraumatic stress disorder. Arch Gen Psychiatry 2003;60:1024-32.

What is the most effective early intervention for people with post-traumatic stress disorder (PTSD): cognitive therapy, provision of a self help booklet, or repeated assessments?

METHODS

(

Design: Randomised controlled trial.

$\searrow$

Allocation: Concealed.

Blinding: Assessors blinded to treatment.

Follow up period: Nine months.

Setting: John Radcliffe Hospital, Oxford, and the Northampton General Hospital, Northampton, UK; February 1998 to January 2001

Patients: 85 people developing PTSD within 3 months of a motor vehicle accident (MVA). Inclusion criteria: aged 18-65 years; diagnosis of PTSD (DSM-IV); a score of $\geqslant 20$ on the posttraumatic diagnosis scale (PDS), and intervention commencing within 6 months of the MVA. Exclusion criteria: unconsciousness for > 15 minutes after the MVA or no memory of the accident; history of psychosis or current substance addiction; borderline personality disorder, or severe depression.

$\mathbf{R}_{\mathbf{X}}$

Intervention: Participants not recovering after a 3 week self monitoring phase were randomised to: cognitive therapy (maximum of 12 weekly sessions for 3 months and up to 3 monthly booster sessions); self help booklet ( 64 page self help booklet, customised for people experiencing an MVA, plus educational and motivational support from a clinician); repeated assessments (20 minute session with a clinician followed by regular monitoring and assessment).

Outcomes: Change in the severity of PTSD symptoms, assessed by participants (PDS) and clinicians (clinician administered PTSD scale (CAPS)).

a Patient follow up: $93 \%$.

\section{MAIN RESULTS}

At 9 months, cognitive therapy was more effective at reducing PTSD compared with the self help booklet $(\mathrm{p}<0.001)$ and repeated assessment $(\mathrm{p}<0.001$; see table $)$.

\section{CONCLUSIONS}

Cognitive therapy is more effective at reducing recent onset PTSD compared with either provision of a self help booklet or repeated assessments.
Table Mean PTSD symptom measures before randomisation and 9 months after intervention for people completing the intervention

\begin{tabular}{|c|c|c|c|c|c|c|}
\hline & \multicolumn{2}{|c|}{$\begin{array}{l}\text { Cognitive } \\
\text { therapy } \\
(n=28 ; 100 \%)\end{array}$} & \multicolumn{2}{|c|}{$\begin{array}{l}\text { Self help } \\
\text { booklet } \\
\text { ( } n=25 ; 89 \% \text { ) }\end{array}$} & \multicolumn{2}{|c|}{$\begin{array}{l}\text { Repeated } \\
\text { assessments } \\
(\mathrm{n}=27 ; 93 \% \text { ) }\end{array}$} \\
\hline & $\begin{array}{l}\text { After self } \\
\text { monitoring }\end{array}$ & $\begin{array}{l}9 \\
\text { months }\end{array}$ & $\begin{array}{l}\text { After self } \\
5 \text { monitoring }\end{array}$ & $\begin{array}{l}9 \\
\text { months }\end{array}$ & $\begin{array}{l}\text { After self } \\
\text { monitoring }\end{array}$ & $\begin{array}{l}9 \\
\text { months }\end{array}$ \\
\hline \multicolumn{7}{|l|}{$\begin{array}{l}\text { PDS self } \\
\text { report }\end{array}$} \\
\hline Frequency & 26.2 & 8.7 & 27.9 & 20.0 & 27.0 & 19.4 \\
\hline Distress & 25.8 & 7.3 & 27.3 & 19.0 & 26.2 & 20.0 \\
\hline \multicolumn{7}{|l|}{$\begin{array}{l}\text { CAPS } \\
\text { assessor }\end{array}$} \\
\hline Frequency & y 31.7 & 10.2 & 32.6 & 21.4 & 32.8 & 21.1 \\
\hline Intensity & 26.7 & 9.7 & 26.7 & 18.6 & 25.9 & 17.0 \\
\hline
\end{tabular}

\section{Commentary}

The question of how best to help individuals avoid the development of chronic post-traumatic stress disorder (PTSD) has been increasingly studied. Early single session interventions for all individuals exposed to the trauma do not seem to help ; however, brief (four or five sessions) cognitive behavioural interventions for symptomatic individuals started within six weeks of the trauma do. ${ }^{2} 3$ This study concerns a longer $(11$ sessions on average) cognitive therapy intervention for individuals with PTSD starting on average four months after the traumatic event.

The standard of methodological rigour coupled with the excellent retention rates (94\% post treatment and $93 \%$ at follow up) make it likely that the results of this study are valid. The cognitive therapy technique used was trauma focused, but placed a greater emphasis on cognitive techniques than many of the earlier, more exposure based interventions. The improvement in the cognitive therapy group is impressive and strongly suggests that the intervention is effective. However, as with all studies - and particularly those conducted by the authors of a specific treatment protocol-it will be interesting to see if the results can be replicated by other centres.

The failure of the self help booklet group to improve more than the repeated assessments group is important and, like other studies, ${ }^{1}$ suggests that very limited input is no more helpful than no specific input at all. On the surface this seems to challenge the notion that to give educational information is always beneficial, and suggests that more research is needed regarding this before accepting it as part of routine clinical practice.

This study adds to the existing evidence base that suggests that the best way to prevent chronic PTSD is to identify individuals with traumatic stress symptoms within a few months of a traumatic event and to offer them a brief cognitive behavioural intervention.

Jonathan I Bisson, DM MRCPsych
Vale NHS Trust, Cardiff, UK
Consultant Liaison Psychiatrist and Honorary Senior Lecturer, Cardiff \&
I Rose S, Bisson J, Wessely S. Brief psychological interventions ("debriefing")
for trauma-related symptoms and prevention of post traumatic stress
disorder (Cochrane Review). In: The Cochrane Library, Issue 1. Oxford:
Update Software, 2003.
2 Bryant RA, Sackville T, Dang ST, et al. Treating acute stress disorder: an
evaluation of cognitive behavior therapy and supportive counseling
techniques. Am J Psychiatry 1999;156:1780-6.
3 Bisson JI, Shepherd JP, Joy D, et al. Early cognitive-behavioural therapy for
post-traumatic stress symptoms after physical injury. Randomised controlled
trial. Br J Psychiatry 2004;184:63-9.

For correspondence: Dr A Ehlers, Department of Psychology, Institute of Psychiatry, De Crespigny Park, Denmark Hill, London, UK; a.ehlers@ iop.kcl.ac.uk

Sources of funding: The Wellcome Trust, London, UK, The Oxfordshire NHS Trust Research and Development Fund, Oxford, UK. 